\title{
SCHWANOMA PARAFARINGEO
}

\author{
PARAPHARYNGEAL SCHAWANNNOMA \\ Enrique Moyano Navarro1,a, Manuel Inostroza Fernández ${ }^{1, a}$, Pedro Sotelo Jiménez ${ }^{1, a}$, Ethel Vargas Carrillo², , \\ Alan La Torre Zúñiga ${ }^{1, c}$, Luis González Domínguez ${ }^{1, d}$
}

\begin{abstract}
RESUMEN
Las lesiones tumorales de localización parafaríngea presentan una escasa incidencia, representando entre el 0,5 y el $0,8 \%$ de todas las tumoraciones de cabeza y cuello. Aproximadamente el $80 \%$ presentan un comportamiento benigno. La singularidad de dichas lesiones deriva de su compleja situación anatómica y de la clínica con la que suelen debutar, siendo en la mayoría de los casos inespecífica y casi siempre derivada del efecto compresivo producido por la lesión sobre la oro y rinofaringe. Los Schwannoma del espacio parafaríngeo son tumores muy infrecuentes, que se originan de la vaina de schawnn, generalmente de crecimiento lento, suelen ser asintomáticos. El tratamiento es quirúrgico, muchas veces complejo por la localización anatómica. Se presenta el caso de una paciente femenina de 42 años de edad con diagnóstico de Schwannoma en la región parafaríngea izquierda demostrada mediante biopsia incisional; realizándosele tratamiento quirúrgico, el cual se llevó a cabo sin complicaciones.
\end{abstract}

Palabras clave: Espacio parafaríngeo; Schwannoma (fuente: DeCS BIREME).

\begin{abstract}
Parapharyngeal tumor lesions present a low incidence, representing between 0,5 and $0,8 \%$ of all head and neck tumors. Approximately $80 \%$ show benign behavior. The uniqueness of these lesions derives from their complex anatomical situation and the symptoms with which they usually appear, being in most cases nonspecific and almost always derived from the compressive effect produced by the lesion on the oropharynx and the oropharynx. Schwannomas of the parapharyngeal space are very rare tumors that originate from the sheath of schawnn, generally slow growing, and are usually asymptomatic. Treatment is surgical, often complex due to the anatomical location. The case of a 42-year-old female patient with a diagnosis of Schwannoma in the left parapharyngeal region, demonstrated by incisional biopsy, is presented; undergoing surgical treatment, which was carried out without complications.
\end{abstract}

Key words: Parapharyngeal space; Schwannoma (source: MeSH NLM).

\footnotetext{
${ }^{1}$ Hospital Nacional Edgardo Rebagliati Martins - Essalud - Lima- Perú.

a Médico Asistente de Medicina Interna.

${ }^{b}$ Médico Residente de Medicina Interna.

'Médico Asistente de Anatomía Patológica.

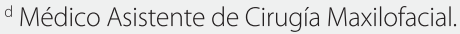

Citar como: Enrique Moyano Navarro, Manuel Inostroza Fernández, Pedro Sotelo Jiménez, Ethel Vargas Carrillo, Alan La Torre Zúñiga, Luis González Domínguez. Schwanoma parafaringeo. Rev. Fac. Med. Hum. Octubre 2021; 21(4):896-902.DOI 10.25176/RFMH.v21i4.3985 


\section{INTRODUCCIÓN}

Los Schwannomas son los segundos tumores en frecuencia de este espacio, aunque la mayoría de estos tumores se localizan en cabeza y cuello, los de localización extracraneal solo constituyen un $10 \%$ del total, de ahí su infrecuente presentación. Las lesiones tumorales de localización parafaríngea presentan una escasa incidencia, representando entre el 0,5 y el $0,8 \%$ de todas las tumoraciones de cabeza y cuello ${ }^{(1,2)}$. Aproximadamente el $80 \%$ presentan un comportamiento benigno ${ }^{(2-4)}$. La singularidad de dichas lesiones deriva de su compleja situación anatómica y de la clínica con la que suelen debutar, siendo en la mayoría de los casos inespecífica y casi siempre derivada del efecto compresivo producido por la lesión sobre la oro y rinofaringe, presentando síntomas como disfonía, dolor compresivo o tumoración para faríngea. El paraganglioma secretor, aunque poco común, puede causar hipertensión, diaforesis, náuseas, palpitaciones 0 cefalea ${ }^{(3)}$ Habitualmente son tumores que afectan a adultos, sin diferencias entre sexos y mayoritariamente son benignos.

El estudio anatomopatológico del tumor revela una masa bien encapsulada constituida por células fusiformes tipo A de Anoni y tipo B de Antoni, la inmunohistoquímica muestran una intensa expresión de la proteína S-100(5).

El pronóstico del Schwannoma es excelente cuando la tumoración ha sido completamente extirpada. Es infrecuente la recidiva local, relacionándose con la exégesis incompleta de la neoplasia ${ }^{(6,7)}$.

\section{CASO CLÍNICO}

Paciente femenina de 42 años de edad, con antecedente de migraña desde hace 20 años, Se presentó al servicio de Medicina Interna del Hospital Edgardo Rebagliati Martins de Lima, Perú, refiriendo un tiempo de enfermedad de 2 meses de inicio progresivo y caracterizado por cefalea hemicraneal y mandibular izquierda, de moderada intensidad, acompañado de aumento de volumen en región cervical del mismo lado; además leve odinofagia y disfonía. Al examen físico evidenció masa de aproximadamente $5 \mathrm{~cm}$ de diámetro en región maxilar izquierda, no adherida a planos profundos, con piel circundante no hipertérmica y sin cambios de coloración, ni fija a planos profundos, a la manipulación bimanual se presenta discretamente dolorosa a la palpación superficial y profunda. Ante la sospecha de proceso expansivo parafaringeo se realizó un estudio de imagen mediante una Tomografía Cervical con contraste (Figura 1), donde se evidencia gran masa solida parafaríngea izquierda con realce heterogéneo del contraste y áreas de calcificación periférica mide DT $38 \mathrm{~mm}$ DL $50 \mathrm{~mm}$ DAP $40 \mathrm{~mm}$, y que se extiende cranealmente hacia el foramen yugular y desplaza anteriormente a las estructuras vasculares cervicales, sin infiltración; ocasiona efecto de masa con compresión de vía aérea. Además, se realizó el estudio de resonancia magnética nuclear (Figura 2, Figura 3), donde muestra una imagen sólida de bordes bien definidos, forma ovoidea en el espacio parafaringeo izquierdo, ejerciendo efecto expnasivo y desplazando la línea media de izquierda a derecha. No se observa extensión intramedular. Posteriormente se realizó una BAAF con guía ecográfica de la lesión cuyo resultado no fue concluyente para el diagnóstico, motivo por el cual caso fue reevaluado, decidiéndose la toma de una biopsia incisional por el Servicio de Cirugía de Cabeza y Cuello, la cual fue enviada a estudio histopatológico; se reportó Schwanoma, siendo transferida para tratamiento quirúrgico definitivo; se utilizó un abordaje cervical (Figura 4, Figura 5), y se envió la pieza quirúrgica a estudio histopatológico, cuyo resultado ratificó nuestro diagnóstico inicial: Schwannoma parafaringeo. 


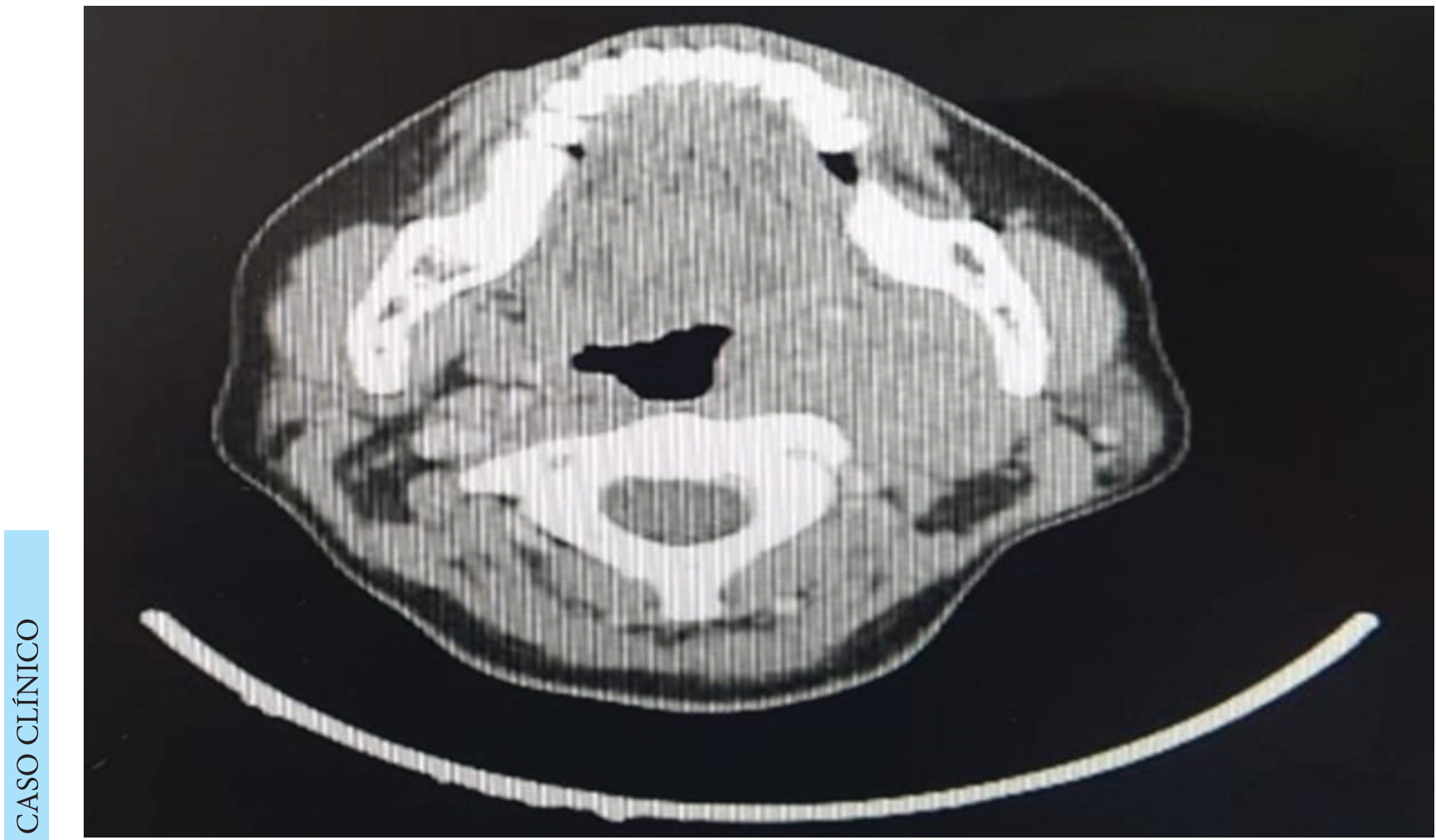

Figura 1. Tomografía de cuello contrastada, Se evidencia gran masa solida parafaríngea izquierda con realce heterogéneo del contraste y áreas de calcificación periférica mide DT 38mm DL 50mm DAP 40 mm, que se extiende cranealmente hacia el foramen yugular y desplaza anteriormente a las estructuras vasculares cervicales, sin infiltración; ocasiona efecto de masa con compresión de vía aérea.

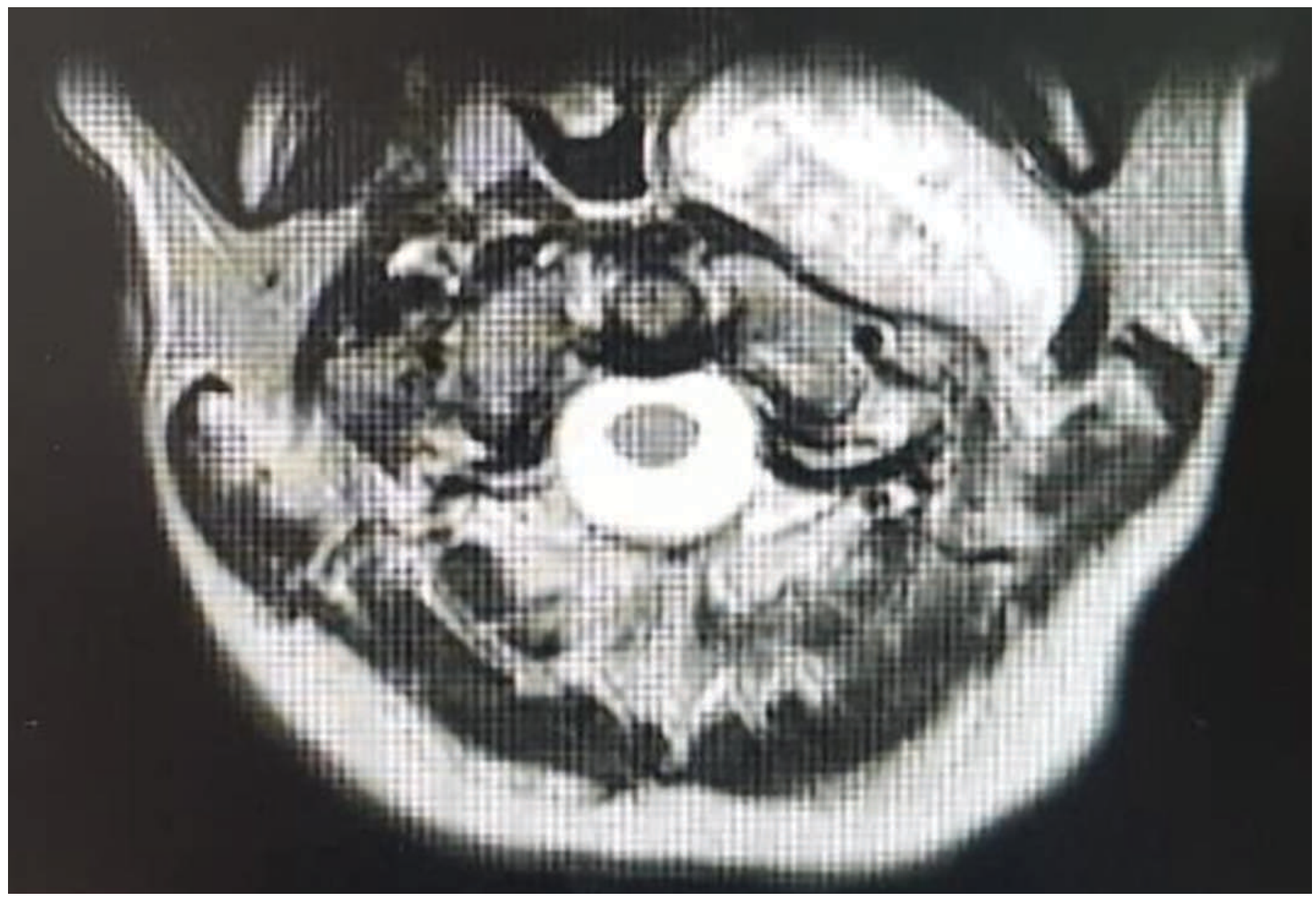

Figura 2. RMN de cuello con contraste, muestra imagen sólida de bordes bien definidos, forma ovoidea en el espacio parafaringeo izquierdo, ejerciendo efecto ocupativo y desplazando la línea media de izquierda a derecha. No se observa extensión intramedular. 


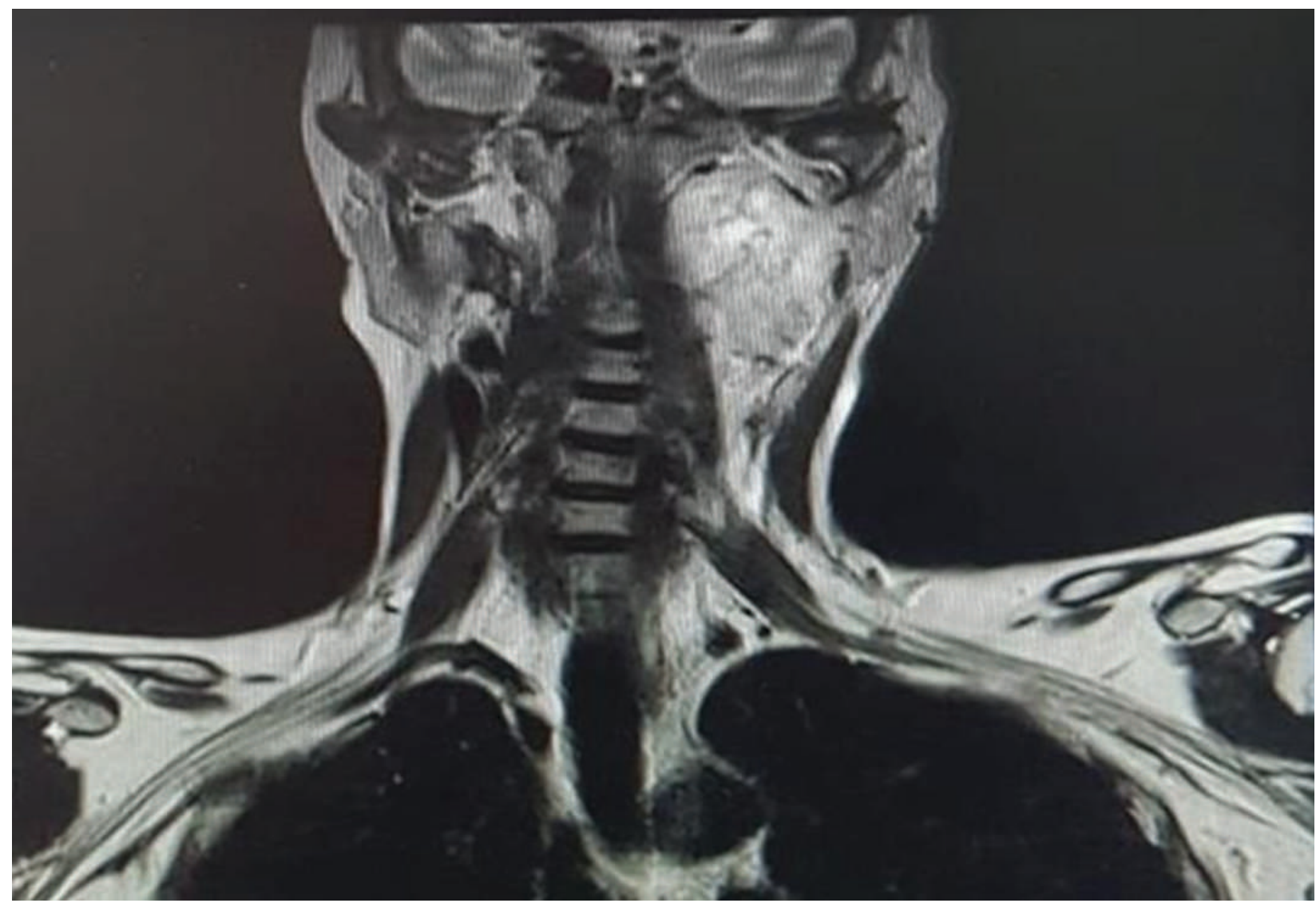

Figura 3. Toma de una biopsia incisional, se utilizó un abordaje cervical por el Servicio de Cirugía de Cabeza y Cuello.



Figura 4. A menor aumento, se aprecia tumor bien delimitado y encapsulado, ya a este aumento se aprecia la presencia de núcleos dispuestos en empalizada (cuerpos de Verocay). 


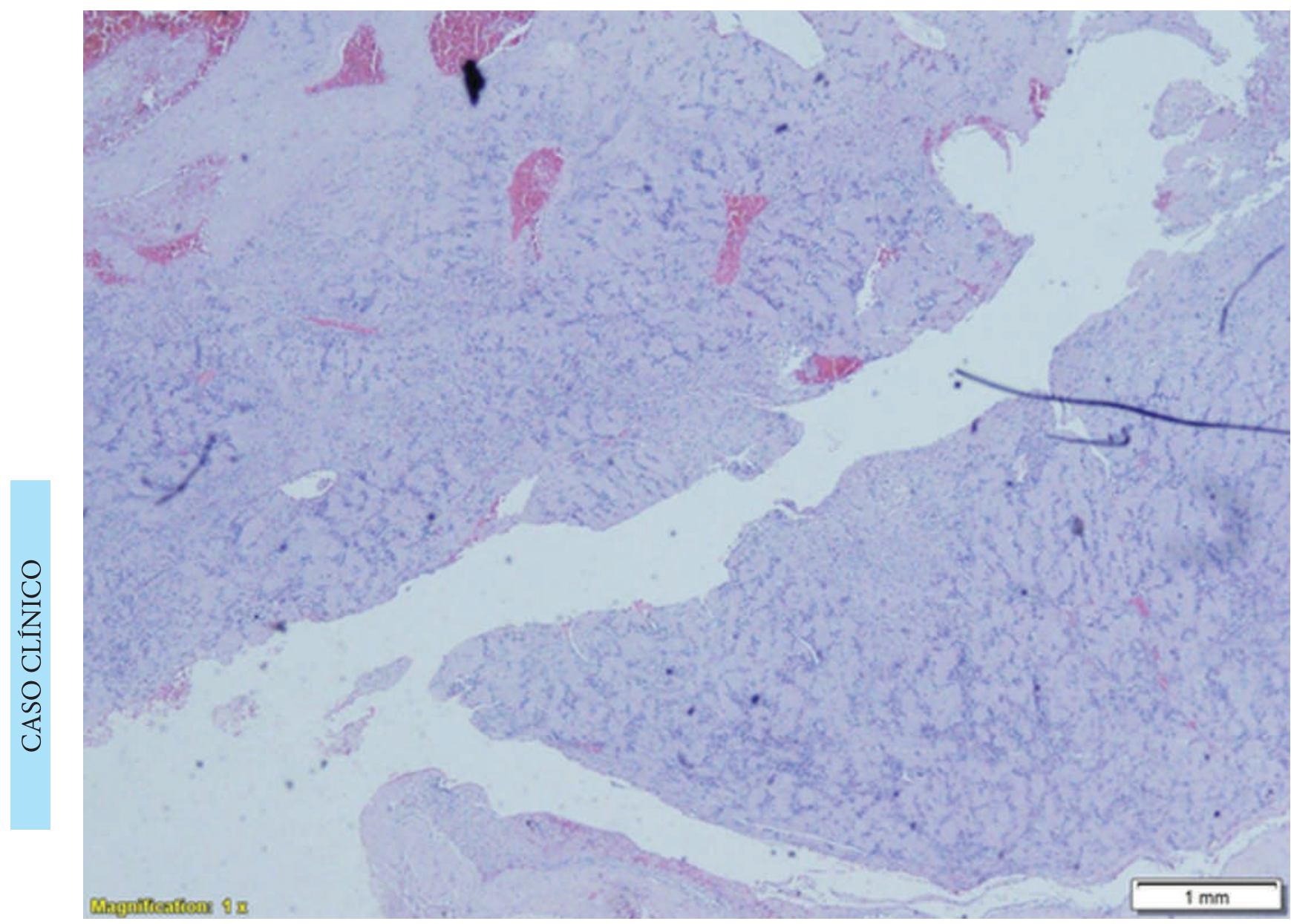

Figura 5. Se aprecia además de los cuerpos de Verocay, dilatación vascular, otro hallazgo frecuente en Schwannomas.

\section{DISCUSIÓN}

Los tumores del espacio parafaríngeo suponen el $0,5 \%$ de todas las neoplasias de cabeza y cuello(2). La mayoría de las lesiones localizadas en este espacio son tumores primarios benignos que se originan del nervio vago, siendo el schwannoma el más común. El schwannoma es el subtipo histológico más frecuente de tumoración en el nervio vago después del paraganglioma. Suele aparecer entre la tercera y la sexta décadas de la vida, siendo más frecuente en el adulto joven ${ }^{(5)}$, presentando una frecuencia similar en ambos sexos ${ }^{(3)}$. Clínicamente asintomático, se diagnostica como una masa unilateral laterocervical móvil únicamente en dirección lateral solitaria, salvo en la enfermedad de Von Recklinghausen ${ }^{(6,8)}$ y en un raro síndrome conocido como schwannomatosis ${ }^{(24)}$ donde la forma de presentación es múltiple. Pueden palparse como masas en el borde medial del músculo esternocleidomastoideo. Su crecimiento expansivo por el espacio parafaríngeo puede hacer que pase desapercibido hasta alcanzar un gran tamaño. Si su tamaño es considerable puede provocar signos muy inespecíficos como parestesias faríngeas, tos, odinofagia por compresión faríngea, disfonía. Un signo clínico característico, aunque no siempre presente, del schwannoma vagal cervical es la tos paroxística al palpar la tumoración e incluso otitis serosa ${ }^{(5,6,9)}$. Los síntomas derivados de compresión de estructuras nerviosas pares craneales IX, X, XI, XII, del simpático se verán en casos de neurinomas de gran tamaño, o bien en tumores malignos y quemodectomas. Dolor, trismus y lesiones de pares craneales sugieren malignidad.

El diagnostico se basará en la clínica, la exploración física, siendo importante la palpación bimanual, que permite reconocer la continuidad del tumor y determinar su origen. Es importante la visualización orofaríngea y de la cavidad oral para descartar otros primarios de esas localizaciones que pudieran estar produciendo un compromiso secundario metastásico en el espacio parafaríngeo. Una masa pulsátil cervical puede orientar a la presencia de un tumor vascular, como un paraganglioma. El examen físico debe incluir examen de pares craneanos y 
la realización de una nasofaringolaringoscopía flexible ${ }^{(2)}$.

Será de vital importancia el estudio radiológico mediante Tomografía Computarizada (TC) y Resonancia Magnética (RM). En la TAC son tumores de densidad similar al músculo, más menos redondeados bien definidos con plano graso medial y lateral, como su origen más frecuente es el X par - la cadena simpática, suelen desplazar la arteria carótida interna anteromedialmente. La RM aporta una excelente información en el estudio de las partes blandas, siendo muy útil en este tipo de tumores y dicha región,

son tumores hipointensos en T1 y hiperintensos en T2 y en T1 con gadolinio. Se debe realizar diagnóstico diferencial con el aneurisma de la arteria carótida, con los tumores neurogénicos de los pares craneales IX, X, XI y XII o del plexo simpático cervical| ${ }^{(14)}$, con el paraganglioma, el meningioma, el linfoma y el teratoma ${ }^{(15)}$. Sin embargo, la mayoría de lesiones que involucran al espacio parafaríngeo retroestiloideo son tumores de la vaina de los nervios periféricos, paragangliomas de cabeza y cuello o linfadenopatías metastásicas $^{(24)}$.

El rendimiento de la PAAF en los tumores del espacio pararafingeo puede llegar al 86\%- 99\% especialmente en linfoma, tumores malignos primarios o metástasis de tumores malignos ${ }^{(22,24)}$. Sin embargo, la PAAF no sería de utilidad para distinguir paragangliomas benignos de malignos ni tumores de nervio periférico benignos de malignos. Como en nuestro caso que no fue aporte para diagnóstico.

Contribuciones de autoría: Los autores participaron en la génesis de la idea, diseño de proyecto, recolección e interpretación de datos, análisis de resultados y preparación del manuscrito del presente reporte de caso.

Financiamiento: Autofinanciado.

Correspondencia: Javier Enrique Moyano Navarro

Dirección: Av. Rebagliati s/n - Jesús María-Lima-Perú

Teléfono: 999025042

Correo:enriquemoyano_ica@hotmail.com
El examen macroscópico suele corresponder a una tumoración de color blanca, amarillenta, bien delimitada de consistencia firme y elástica, en ocasiones con áreas quísticas. siendo típica la presencia de necrosis, hemorragia y degeneración quística intratumoral(23). Desde el punto de vista microscópico existen dos grupos: Antoni A composición celular compacta en empalizada y Antoni B formado por una estructura laxa que se caracteriza por una degeneración mixoide, aunque la mayoría de las veces se presentan asociados, mientras que otros defienden que la hemorragia es responsabledela degeneración vacuolarpordepósito de hemosiderina. Las técnicas inmunohistoquímicas muestran una intensa expresión de la proteína S-100 en estos tumores, un antígeno de los tejidos derivados de la cresta neural ${ }^{(20,21)}$.

El tratamiento es quirúrgico, aunque debido a la infrecuente presentación de tumores en dicha localización es difícil la obtención de experiencia quirúrgica, existen diversas vías de abordaje, la cual estará en función del tamaño y la localización del tumor, siendo la más utilizada la vía cervical, adecuada para la mayoría de tumores, en casos de mayor tamaño o bien para tumores de localización preestilea puede ser útil el abordaje cérvico-parotideo. Las complicaciones más frecuentes en la cirugía son la lesión del propio nervio y su déficit resultante, las recurrencias son raras y suelen ser resultantes de una exéresis incompleta, teniendo en general un buen pronóstico postquirúrgico. Respecto al pronóstico, en casos de tumores benignos, cuando se logra una resección completa el riesgo de recurrencia debería ser bajo a nulo.

Conflicto de interés: Los autores declaran no tener conflicto de interés.

Recibido: 19 de julio 2021

Aprobado: 18 agosto 2021 


\section{REFERENCIAS BIBLIOGRÁFICAS}

1. Peter Pang K, Goh C, Ming Tan H. Parapharyngeal space tumours: an 18 year review. J Laryngol Otol 2002;116:170-5.

2. Olsen KD. Tumors and surgery of the parapharyngeal space. Laryngoscope 1994;104 (suppl 63).

3. Lawson VG, LeLiever WC, Makerewich LA. Unusual parapharyngeal lesions. J Otolaryngol 1979;8:241-9.

4. Andratschke M, Helmberguer R, Mess K. Differential diagnosis of parapharyngeal mass. Laryngorhinootologie 2000;79:174-9.

5. Asano K, Kubo O, Tajika Y, Ishii T, Tanikawa T, Kawamura H, et al. A clinico- pathological study of cystic spinal schwannomas. No To Shinkei 1996; 48: 245-51.

6. Donnelly MJ, AI-Sader MH, Blaney AW. View from beneath: Pathology in focus. Benign nasal schwannoma. J Laryngol Otol 1992; 106: 1011-5.

7. Nao EE, Dassonville O, Bozec A, Sudaka A, Marcy PY, Vincent N, et al. Cervical sympathetic chain schwannoma. Eur Ann Otorhinolaryngol Head Neck Dis 2012; 129: 51-3.

8. Najib Benmansour, Yasine Elfadl, Amal Bennani, Mustapha Maaroufi, Leila Chbani, Afaf Amarti, et al. Schwannome cervical du nerf vague: Stratégies diagnostique et thérapeutique. Pan Afr Med J 2013; 14: 76
9. Chiofalo MG, Longo F, Marone U, Franco R, Petrillo A, Pezzullo L. Cervical vagal schwannoma. A case report. Acta Otorhinolaryngol Ital 2009; 29: 33-35.

10. De Araujo CE, Ramos DM, Moyses RA, Durazzo MD, Cernea $C R$, Ferraz AR. Neck nerve trunks schwannomas: clinical features and postoperative neurologic outcome. Laryngoscope 2008; 118: 1579-82.

11. Colreavy MP, Lacy PD, Hughes J, Bouchier-Hayes D, Brennan P, O’Dwyer AJ, et al. Head and neck schwannomas - a 10-year review. J Laryngol Otol 2000; 114: 119-24.

12. Ríos A, Rodríguez JM, Febrero B, Parrilla P. Schwannoma extracraneal de origen simpático cervical. Rev Neurol 2012; 54: 638-9.

13. Gilmer-Hill HS, Kline DG. Neurogenic tumours of the cervical vagus nerve: report of four cases and review of the literature. Neurosurgery 2000; 46: 1498-503.

14. García-Marín A, Martín-Gil J, Bernardos-García LE, Gómez-Lanz L, Turégano- Fuentes F. Cervical cystic schwannoma of the vagus nerve. Cir Esp 2011; 89: 627- 8

15. Varoquaux A, Fakhry N, Gabriel S, Garcia S, Ferretti A, Chondrogiannis $S$, et al. Retrostyloid parapharyngeal space tumors: a clinician and imaging perspective. Eur J Radiol 2013; 82: 773-82.

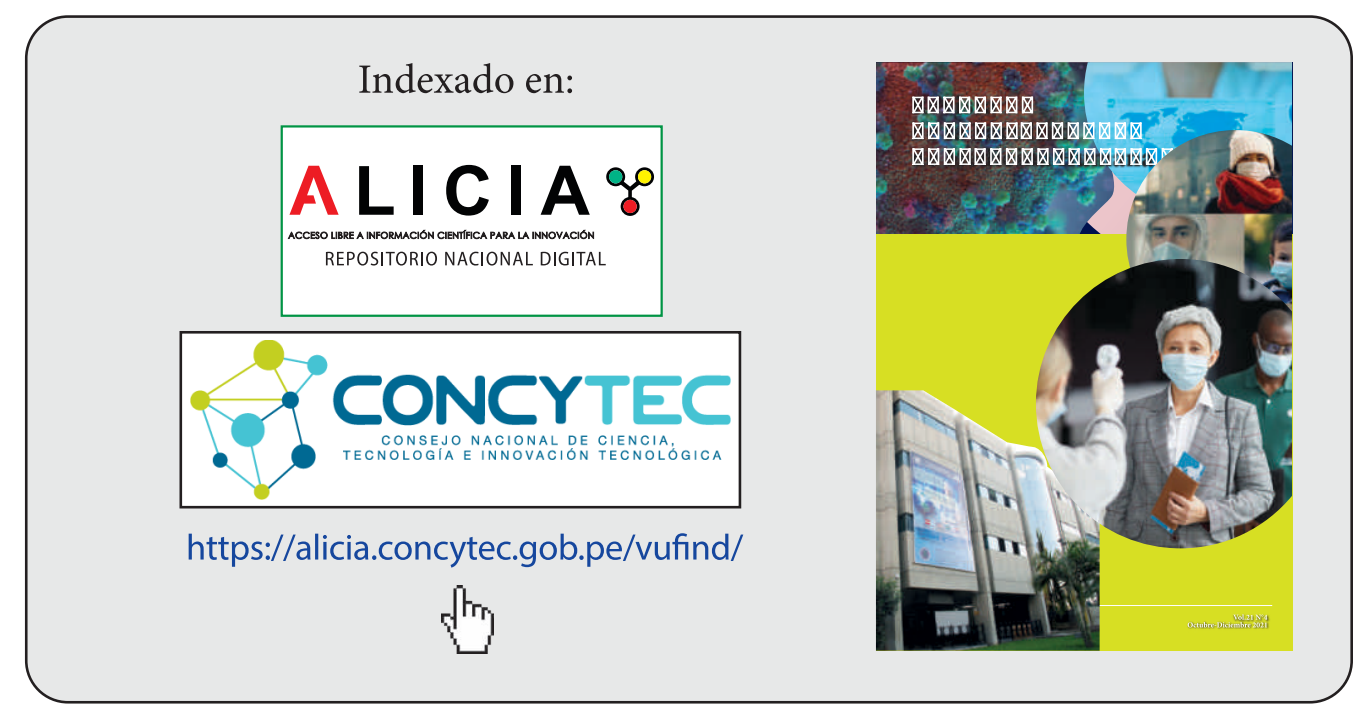

